\title{
Power Spectrum Equalized Scalar Representation of Wide-Angle Optical Field Propagation
}

\author{
Onur Kulce $^{1}$ (D) $\cdot$ Levent Onural ${ }^{1}$ \\ Received: 30 May 2017 / Accepted: 30 March 2018 / Published online: 6 April 2018 \\ (c) Springer Science+Business Media, LLC, part of Springer Nature 2018
}

\begin{abstract}
It may be desirable to represent optical fields using scalar approximations, due to its simplicity. Since the optical field is an electromagnetic wave, in order to implement an optical setup, a mapping from such a scalar field to the vector electromagnetic field is needed. In the conventional scalar-to-vector field mapping, a large error in power spectrum arises in wide-angle fields due to the neglected large longitudinal component of the electric field. This error could be severe in wide-angle or off-axis imaging setups. In order to find another scalar-to-vector field mapping that compensates for this large magnitude error, first, a general constraint on monochromatic electromagnetic fields to appropriately represent them by a scalar wave in free space is developed. The development of the general constraint begins by formulating the computations of the components of the magnetic field as the outputs of linear-shift invariant (LSI) systems, where the inputs to the LSI systems are the transverse components of the electric field. Furthermore, if one of the transverse components of the electric field can be computed from the other one using a LSI operation, a scalar field, which is related to the transverse components through another LSI system, can be used to fully describe the electromagnetic field. Under this constraint, the required condition on the filters which relates the scalar field to the electric field is presented by taking into consideration the longitudinal component, such that the power spectra of the scalar field and the corresponding electric field are equal. The filters are specified for the electric fields with zero longitudinal component and simple polarization features, as well. Moreover, for the electric fields with simple polarization features, some discrete simulations are performed to compare the scalar field intensity pattern and the corresponding electric field intensity patterns for the conventional and proposed mapping cases. The simulation results show that the excessive amplification of the large frequency components is compensated by the proposed filters, and hence, the undesired effects of the filters used in the computation of the longitudinal component disappear. In this respect, if equality of the power spectra of the scalar field and the corresponding electric field is of concern in an application, the proposed scalar-to-vector wave field mapping should be used.
\end{abstract}

Keywords Wave propagation · Power spectrum of optical fields · Inverse filtering · Imaging and display of wide-angle fields

\section{Introduction}

Scalar wave theory may be used to explain some wave-related features of light, such as interference and diffraction [5,14]. Many optical systems including imaging systems, communication systems and holography have been analyzed and developed using the scalar theory $[12,14,19,30]$. During the implementation of an optical system that is characterized by

Onur Kulce acknowledges partial support of TÜBİTAK for this work in the form of a scholarship.

\footnotetext{
Onur Kulce

kulce@ee.bilkent.edu.tr

1 Bilkent University, Ankara, Turkey
}

the scalar theory, the mapping of the scalar results to the vector electromagnetic case is inevitable since the optical field is an electromagnetic wave. When the field is paraxial, the longitudinal component of the electromagnetic field becomes negligible $[17,18]$, and hence, the scalar field can be mapped to one of the transverse components of the electric field if there is a simple polarization relation between the transverse components of the electric field, i.e., one transverse component is a constant multiple of the other one [4,30]. This conventional scalar-to-vector field mapping is performed in many display applications by assuming that the electric field is linearly or circularly polarized [13,20,23,25,31,32]. However, if the field is a wide-angle field, the longitudinal component cannot be neglected and the actual power spec- 
trum of the field may be significantly different from the power spectrum predicted by the conventional scalar theory [18]. As a consequence of such an approach, implemented optical system may not generate the intended results; the obtained power spectrum may significantly deviate from the desired results.

In this paper, we develop and specify another scalar-tovector wave field mapping for wide-angle fields such that the power spectra of the scalar field and the corresponding electric field are equal. In order to accomplish this, we first derive a general constraint on monochromatic electromagnetic wave fields in free space such that they can be appropriately represented by a scalar wave field. This general constraint turns out to be an operation between the transverse components of the electric field so that one of the transverse components can be computed from the other one using a predetermined linear-shift invariant (LSI) system which satisfies given properties. Under this constraint, we develop a condition for LSI filters that are used to map the scalar wave field to the vector wave field such that the resulting power spectra of the scalar field and the electric field are equal.

The developed scalar-to-vector field mapping can be used in different imaging applications. For example, in a nonparaxial imaging system where the electric field intensity is recorded, the deteriorations which occur due to the excessive amplification of the longitudinal component in large angles are compensated by the presented power spectrum equalization procedure. Moreover, in an off-axis optical imaging setup where the magnitude of the two-dimensional (2D) Fourier transform (FT) of the field at a tilted and rotated plane is recorded, if the electromagnetic field is generated using the proposed method, then the intended scalar results can be obtained without error.

In Sect. 2, we present the preliminary information, and in Sect. 3, we express the computations of the components of the magnetic field from the transverse components of the electric field both in the Fourier and in space domains and for both propagating only and general fields. Then, in Sect. 4, a general constraint on the full representation of electromagnetic wave fields as a scalar wave will be shown. Based on these constraints, in Sect. 5, a power spectrum equalizing LSI transform is developed. In Sect. 6, we present some discrete simulation results that compare the performances of the conventional and proposed scalar-to-vector wave field mappings. Finally in Sect. 7, we draw the conclusions.

\section{Preliminaries}

Since we assume that the electromagnetic wave field is monochromatic, the time dependency of the complex-valued field, $e^{-j \omega t}$, is omitted throughout this article, where $j=$ $\sqrt{-1}, t$ is the time and $\omega=c k$. Here, $c$ represents the speed of light in free space, $k$ is the wave number and depends on the wavelength, $\lambda$, of the monochromatic field by $k=2 \pi / \lambda$.

Let the electric and magnetic field vectors in three-

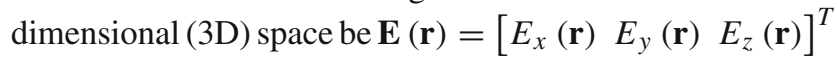
$\in \mathbb{C}^{3}$ and $\mathbf{H}(\mathbf{r})=\left[H_{x}(\mathbf{r}) H_{y}(\mathbf{r}) H_{z}(\mathbf{r})\right]^{T} \in \mathbb{C}^{3}$, respec-

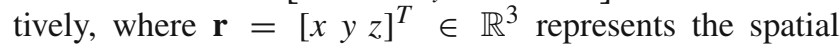
coordinates. We assume that $\mathbf{E}(\mathbf{r})$ and $\mathbf{H}(\mathbf{r})$ can be written as a superposition of plane waves as [2]

$\mathbf{E}(\mathbf{r})=\frac{1}{4 \pi^{2}} \int_{-\infty}^{\infty} \mathcal{E}(\hat{\mathbf{k}}) e^{j k_{z} z} e^{j \hat{\mathbf{k}}^{T} \hat{\mathbf{r}}} \mathrm{d} \hat{\mathbf{k}}$,
$\mathbf{H}(\mathbf{r})=\frac{1}{4 \pi^{2}} \int_{-\infty}^{\infty} \mathcal{H}(\hat{\mathbf{k}}) e^{j k_{z} z} e^{j \hat{\mathbf{k}}^{T} \hat{\mathbf{r}}} \mathrm{d} \hat{\mathbf{k}}$,

where $\hat{\mathbf{k}}=\left[\begin{array}{ll}k_{x} & k_{y}\end{array}\right]^{T} \in \mathbb{R}^{2}$ is the spatial frequency vector and $\hat{\mathbf{r}}=\left[\begin{array}{ll}x & y\end{array}\right]^{T}$. Since $k_{x}$ and $k_{y}$ are always real valued as a consequence of the choice implied by the range of $\hat{\mathbf{k}}$ in Eq. 1, the monochromaticity condition that should be satisfied becomes [2]

$|\hat{\mathbf{k}}|^{2}+k_{z}^{2}=k^{2}$.

By assuming that the propagation direction of the propagating plane waves along the $z$-axis is always positive and the evanescent components decay as $z$ increases, we choose $k_{z}$ as

$k_{z}= \begin{cases}\sqrt{k^{2}-|\hat{\mathbf{k}}|^{2}} & \text { if }|\hat{\mathbf{k}}|<k \\ j \sqrt{|\hat{\mathbf{k}}|^{2}-k^{2}} & \text { otherwise. }\end{cases}$

Please note that the evanescent modes of the field at a given $z$ plane vanish rapidly in 3D space as $z$ increases. That is, their contribution to the $3 \mathrm{D}$ field becomes negligible for practical imaging distances [14].

If the field components at $z=0$ plane are known, then the 2D FT of these field components give the vector amplitude of each plane wave. That is,

$$
\begin{aligned}
\mathcal{E}(\hat{\mathbf{k}}) & =\iint_{-\infty}^{\infty} \mathbf{E}(\hat{\mathbf{r}}, 0) e^{-j \hat{\mathbf{k}}^{T} \hat{\mathbf{r}}} \mathrm{d} \hat{\mathbf{r}}, \\
\mathcal{H}(\hat{\mathbf{k}}) & =\iint_{-\infty}^{\infty} \mathbf{H}(\hat{\mathbf{r}}, 0) e^{-j \hat{\mathbf{k}}^{T} \hat{\mathbf{r}}} \mathrm{d} \hat{\mathbf{r}},
\end{aligned}
$$

where $\mathcal{E}(\hat{\mathbf{k}})=\left[\mathcal{E}_{x}(\hat{\mathbf{k}}) \mathcal{E}_{y}(\hat{\mathbf{k}}) \mathcal{E}_{z}(\hat{\mathbf{k}})\right]^{T} \in \mathbb{C}^{3}$ and $\boldsymbol{H}(\hat{\mathbf{k}})=\left[\mathcal{H}_{x}(\hat{\mathbf{k}}) \mathcal{H}_{y}(\hat{\mathbf{k}}) \mathcal{H}_{z}(\hat{\mathbf{k}})\right]^{T} \in \mathbb{C}^{3}$. 
Therefore, if the electromagnetic field at $z=0$ plane is known, the field at an arbitrary $z$ plane can be found using Eqs. 1 and 4. For the sake of the completeness, we also write these relations as 2D LSI operations in the space domain as [29]

$\mathbf{E}(\hat{\mathbf{r}}, z)=\mathbf{E}(\hat{\mathbf{r}}, 0) * * f_{z}(\hat{\mathbf{r}})$,

$\mathbf{H}(\hat{\mathbf{r}}, z)=\mathbf{H}(\hat{\mathbf{r}}, 0) * * f_{z}(\hat{\mathbf{r}})$,

where $* *$ denotes the $2 \mathrm{D}$ convolution, $f_{z}(\hat{\mathbf{r}})$ is the impulse response of the Rayleigh-Sommerfeld free space propagation and equals to

$f_{z}(\hat{\mathbf{r}})=-\frac{1}{2 \pi} \frac{\partial}{\partial z}\left[\frac{e^{j k|\mathbf{r}|}}{|\mathbf{r}|}\right]=-\frac{e^{j k|\mathbf{r}|}}{2 \pi}\left(j k-\frac{1}{|\mathbf{r}|}\right) \frac{z}{|\mathbf{r}|^{2}}$

which is the 2D inverse FT (IFT) of $\mathcal{F}_{z}(\hat{\mathbf{k}})=e^{j k_{z} z}$.

In free space, the $z$ component of the electric field at a $z$ plane can be computed from the $x$ and $y$ components as $[18,21]$

$E_{z}(\hat{\mathbf{r}}, z)=E_{x}(\hat{\mathbf{r}}, z) * * g_{x}(\hat{\mathbf{r}})+E_{y}(\hat{\mathbf{r}}, z) * * g_{y}(\hat{\mathbf{r}})$,

where

$g_{x}(\hat{\mathbf{r}})=\frac{1}{2 \pi} \frac{\partial}{\partial x}\left[\frac{e^{j k|\hat{\mathbf{r}}|}}{|\hat{\mathbf{r}}|}\right]=\frac{e^{j k|\hat{\mathbf{r}}|}}{2 \pi}\left(j k-\frac{1}{|\hat{\mathbf{r}}|}\right) \frac{x}{|\hat{\mathbf{r}}|^{2}}$,

$g_{y}(\hat{\mathbf{r}})=\frac{1}{2 \pi} \frac{\partial}{\partial y}\left[\frac{e^{j k|\hat{\mathbf{r}}|}}{|\hat{\mathbf{r}}|}\right]=\frac{e^{j k|\hat{\mathbf{r}}|}}{2 \pi}\left(j k-\frac{1}{|\hat{\mathbf{r}}|}\right) \frac{y}{|\hat{\mathbf{r}}|^{2}}$,

which are the 2D IFTs of [18]

$\mathcal{G}_{x}(\hat{\mathbf{k}})=\mathfrak{F}_{2 D}\left\{g_{x}(\hat{\mathbf{r}})\right\}= \begin{cases}-\frac{k_{x}}{k_{z}} & \text { if } k_{z} \neq 0 \\ 0 & \text { if } k_{z}=0,\end{cases}$
$\mathcal{G}_{y}(\hat{\mathbf{k}})=\mathfrak{F}_{2 D}\left\{g_{y}(\hat{\mathbf{r}})\right\}= \begin{cases}-\frac{k_{y}}{k_{z}} & \text { if } k_{z} \neq 0 \\ 0 & \text { if } k_{z}=0,\end{cases}$

respectively, where $\mathfrak{F}_{2 D}\{\cdot\}$ operation denotes the 2D FT. Therefore, Eq. 7 can be seen as a two-input-single-output LSI system. Please note that, since we $\operatorname{set} \mathcal{E}(\hat{\mathbf{k}})$ is zero when $k_{z}=0, \mathcal{G}_{\{x, y\}}(\hat{\mathbf{k}})$ can be taken as zero in this case, as well. Also note that the link between Eqs. 8 and 9 is established by applying the derivative property of the FT to the filter transfer function

$\mathcal{G}_{0}(\hat{\mathbf{k}})=\left\{\begin{array}{l}-\frac{1}{k_{z}} \quad \text { if } k_{z} \neq 0 \\ 0 \quad \text { if } k_{z}=0\end{array}\right.$ and its $2 \mathrm{D}$ IFT is $[8,18]$

$g_{0}(\hat{\mathbf{r}})=\frac{j}{2 \pi} \frac{e^{j k|\hat{\mathbf{r}}|}}{|\hat{\mathbf{r}}|}$

If the electromagnetic field is known to be propagating, i.e., there is no evanescent component, then, $\mathcal{G}_{0}(\hat{\mathbf{k}}), \mathcal{G}_{x}(\hat{\mathbf{k}})$ and $\mathcal{G}_{y}(\hat{\mathbf{k}})$ can be assumed as zero when $|\hat{\mathbf{k}}| \geq k$. In this case, the 2D IFT of these functions become [18]

$g_{0, p}(\hat{\mathbf{r}})=-\frac{\sin (k|\hat{\mathbf{r}}|)}{2 \pi|\hat{\mathbf{r}}|}=\operatorname{Re}\left\{g_{0}(\hat{\mathbf{r}})\right\}$,

$g_{x, p}(\hat{\mathbf{r}})=-j \frac{\partial}{\partial x}\left[g_{0, p}(\hat{\mathbf{r}})\right]=j \operatorname{Im}\left\{g_{x}(\hat{\mathbf{r}})\right\}$,

$g_{y, p}(\hat{\mathbf{r}})=-j \frac{\partial}{\partial y}\left[g_{0, p}(\hat{\mathbf{r}})\right]=j \operatorname{Im}\left\{g_{y}(\hat{\mathbf{r}})\right\}$,

where the subscript $p$ indicates the propagating case and the $\operatorname{Im}\{\cdot\}$ and $\operatorname{Re}\{\cdot\}$ operators give the imaginary and real parts of their inputs, respectively.

As a result, both the free space propagation of the components of the electric field and the computation of the $z$ component from the $x$ and $y$ components of the electric field can be formulated as 2D LSI operations. In the following section, we show that the computation of the three components of the magnetic field, $\mathbf{H}(\mathbf{r})$, can be interpreted as different two-input-single-output LSI systems, as well. Therefore, specifying only the $x$ and $y$ components of the electric field at any $\mathrm{z}$ plane is enough to characterize all components of the electric field in $3 \mathrm{D}$ space.

\section{Systems Formulation of the Computation of the Magnetic Field}

In this section, we formulate the computation of the magnetic field vector from a system point of view. The inputs to the system are $E_{x}(\hat{\mathbf{r}}, z)$ and $E_{y}(\hat{\mathbf{r}}, z)$, and the outputs are the components of the magnetic field vector at the given $z$ plane. The inputs are fed to different systems for each output, as shown below.

The 2D FT of the components of the magnetic field vector can be computed as $[2,3,26]$

$\begin{aligned} \mathcal{H}_{x}(\hat{\mathbf{k}}) & =\frac{\mathcal{E}_{z}(\hat{\mathbf{k}}) k_{y}-\mathcal{E}_{y}(\hat{\mathbf{k}}) k_{z}}{k \eta}, \\ \mathcal{H}_{y}(\hat{\mathbf{k}}) & =\frac{\mathcal{E}_{x}(\hat{\mathbf{k}}) k_{z}-\mathcal{E}_{z}(\hat{\mathbf{k}}) k_{x}}{k \eta}, \\ \mathcal{H}_{z}(\hat{\mathbf{k}}) & =\frac{\mathcal{E}_{y}(\hat{\mathbf{k}}) k_{x}-\mathcal{E}_{x}(\hat{\mathbf{k}}) k_{y}}{k \eta},\end{aligned}$ 
where $\eta$ is the wave impedance of the medium and approximately equal to $120 \pi \mathrm{ohms}$ in free space [2]. By using $\mathcal{G}_{x}(\hat{\mathbf{k}})$ and $\mathcal{G}_{y}(\hat{\mathbf{k}})$, Eq. 13 can be written as

$$
\begin{aligned}
\mathcal{H}_{x}(\hat{\mathbf{k}}) & =\frac{\left[\mathcal{E}_{x}(\hat{\mathbf{k}}) \mathcal{G}_{x}(\hat{\mathbf{k}})+\mathcal{E}_{y}(\hat{\mathbf{k}}) \mathcal{G}_{y}(\hat{\mathbf{k}})\right] k_{y}-\mathcal{E}_{y}(\hat{\mathbf{k}}) k_{z}}{k \eta} \\
& = \begin{cases}\mathcal{E}_{x}(\hat{\mathbf{k}}) \frac{-k_{x} k_{y}}{k \eta k_{z}}+\mathcal{E}_{y}(\hat{\mathbf{k}}) \frac{-k^{2}+k_{x}^{2}}{k \eta k_{z}} & \text { if } k_{z} \neq 0 \\
0 & \text { if } k_{z}=0\end{cases} \\
& =\mathcal{E}_{x}(\hat{\mathbf{k}}) \mathcal{G}_{x x}(\hat{\mathbf{k}})+\mathcal{E}_{y}(\hat{\mathbf{k}}) \mathcal{G}_{x y}(\hat{\mathbf{k}}), \\
\mathcal{H}_{y}(\hat{\mathbf{k}}) & =\frac{\mathcal{E}_{x}(\hat{\mathbf{k}}) k_{z}-\left[\mathcal{E}_{x}(\hat{\mathbf{k}}) \mathcal{G}_{x}(\hat{\mathbf{k}})+\mathcal{E}_{y}(\hat{\mathbf{k}}) \mathcal{G}_{y}(\hat{\mathbf{k}})\right] k_{x}}{k \eta} \\
& = \begin{cases}\mathcal{E}_{x}(\hat{\mathbf{k}}) \frac{k^{2}-k_{y}^{2}}{k \eta k_{z}}+\mathcal{E}_{y}(\hat{\mathbf{k}}) \frac{k_{x} k_{y}}{k \eta k_{z}} & \text { if } k_{z} \neq 0 \\
0 & \text { if } k_{z}=0\end{cases} \\
& =\mathcal{E}_{x}(\hat{\mathbf{k}}) \mathcal{G}_{y x}(\hat{\mathbf{k}})+\mathcal{E}_{y}(\hat{\mathbf{k}}) \mathcal{G}_{y y}(\hat{\mathbf{k}}), \\
\mathcal{H}_{z}(\hat{\mathbf{k}})= & \mathcal{E}_{x}(\hat{\mathbf{k}}) \frac{-k_{y}}{k \eta}+\mathcal{E}_{y}(\hat{\mathbf{k}}) \frac{k_{x}}{k \eta} \\
= & \mathcal{E}_{x}(\hat{\mathbf{k}}) \mathcal{G}_{z x}(\hat{\mathbf{k}})+\mathcal{E}_{y}(\hat{\mathbf{k}}) \mathcal{G}_{z y}(\hat{\mathbf{k}}) .
\end{aligned}
$$

Therefore, Eqs. 14, 15 and 16 represent two-input-singleoutput LSI systems with the transfer functions indicated in the equations as $\mathcal{G}_{x x}(\hat{\mathbf{k}}), \mathcal{G}_{x y}(\hat{\mathbf{k}}), \mathcal{G}_{y x}(\hat{\mathbf{k}}), \mathcal{G}_{y y}(\hat{\mathbf{k}})$, $\mathcal{G}_{z x}(\hat{\mathbf{k}})$ and $\mathcal{G}_{z y}(\hat{\mathbf{k}})$.

In order to compute the components of the magnetic field vector from $E_{x}(\hat{\mathbf{r}}, z)$ and $E_{y}(\hat{\mathbf{r}}, z)$ in the space domain, we calculate the impulse responses of the transfer functions as

$g_{i j}(\hat{\mathbf{r}})=\mathfrak{F}_{2 D}^{-1}\left\{\mathcal{G}_{i j}(\hat{\mathbf{k}})\right\}$ for $i \in\{x, y\}$ and $j \in\{x, y\}$,

where $\mathfrak{F}_{2 D}^{-1}\{\cdot\}$ denotes the 2D IFT. For this purpose, we utilize Eqs. 8, 11 and the derivative property of the FT. So,

$$
\begin{aligned}
g_{x x}(\hat{\mathbf{r}})=-g_{y y}(\hat{\mathbf{r}}) & =\frac{1}{j k \eta} \frac{\partial g_{y}(\hat{\mathbf{r}})}{\partial x}=\frac{1}{j k \eta} \frac{\partial g_{x}(\hat{\mathbf{r}})}{\partial y}, \\
g_{x y}(\hat{\mathbf{r}}) & =-\frac{k}{\eta} g_{0}(\hat{\mathbf{r}})+\frac{j}{k \eta} \frac{\partial g_{x}(\hat{\mathbf{r}})}{\partial x}, \\
g_{y x}(\hat{\mathbf{r}}) & =\frac{k}{\eta} g_{0}(\hat{\mathbf{r}})-\frac{j}{k \eta} \frac{\partial g_{y}(\hat{\mathbf{r}})}{\partial y} .
\end{aligned}
$$

Therefore, from the convolution and derivative properties of the FT, the components of the magnetic field vector in space domain can be written as

$$
\begin{aligned}
& H_{x}(\hat{\mathbf{r}}, z)=E_{x}(\hat{\mathbf{r}}, z) * * g_{x x}(\hat{\mathbf{r}})+E_{y}(\hat{\mathbf{r}}, z) * * g_{x y}(\hat{\mathbf{r}}) \\
& H_{y}(\hat{\mathbf{r}}, z)=E_{x}(\hat{\mathbf{r}}, z) * * g_{y x}(\hat{\mathbf{r}})+E_{y}(\hat{\mathbf{r}}, z) * * g_{y y}(\hat{\mathbf{r}}) \\
& H_{z}(\hat{\mathbf{r}}, z)=\frac{j}{k \eta} \frac{\partial E_{x}(\hat{\mathbf{r}}, z)}{\partial y}-\frac{j}{k \eta} \frac{\partial E_{y}(\hat{\mathbf{r}}, z)}{\partial x} .
\end{aligned}
$$

Please note that, if the field is known to be propagating, then, $g_{0}(\hat{\mathbf{r}}), g_{x}(\hat{\mathbf{r}})$ and $g_{y}(\hat{\mathbf{r}})$ can be replaced by $g_{0, p}(\hat{\mathbf{r}})$, $g_{x, p}(\hat{\mathbf{r}})$ and $g_{y, p}(\hat{\mathbf{r}})$ in Eq. 18.

To sum up, in free space, if the $x$ and $y$ components of the electric field are known over an arbitrary $z$ plane, then all the field components at 3D space can be found through twoinput-single-output 2D LSI systems, which are described in Eqs. 5, 7 and 19.

\section{A General Constraint on the Scalar Representation of Electromagnetic Fields}

In this section, we introduce a new complex-valued 3D scalar function, $S(\mathbf{r})$, to represent a constrained electromagnetic field. We assume that $S(\hat{\mathbf{r}}, z)$ can be computed from $S(\hat{\mathbf{r}}, 0)$ as

$$
S(\hat{\mathbf{r}}, z)=S(\hat{\mathbf{r}}, 0) * * f_{z}(\hat{\mathbf{r}}) .
$$

So, the same free space propagation rule applies to the scalar field $S(\mathbf{r})$ as for the components of the electric and magnetic field vectors. Then we assume that the $x$ and $y$ components of the electric field at $z=0$ plane can be found through some invertible transforms from the scalar field $S(\hat{\mathbf{r}}, 0)$. That is,

$$
E_{x}(\hat{\mathbf{r}}, 0)=\mathfrak{T}_{x}\{S(\hat{\mathbf{r}}, 0)\}, \quad E_{y}(\hat{\mathbf{r}}, 0)=\mathfrak{T}_{y}\{S(\hat{\mathbf{r}}, 0)\},
$$

where $\mathfrak{T}_{x}: \mathbb{C} \rightarrow \mathbb{C}$ and $\mathfrak{T}_{y}: \mathbb{C} \rightarrow \mathbb{C}$ are some one-to-one predetermined operators together with their inverse transforms $\mathfrak{T}_{x}^{-1}$ and $\mathfrak{T}_{y}^{-1}$. So $E_{x}(\hat{\mathbf{r}}, 0)$ and $E_{y}(\hat{\mathbf{r}}, 0)$ are assumed to be related through

$$
\begin{aligned}
& E_{x}(\hat{\mathbf{r}}, 0)=\mathfrak{T}_{x}\left\{\mathfrak{T}_{y}^{-1}\left\{E_{y}(\hat{\mathbf{r}}, 0)\right\}\right\}, \\
& E_{y}(\hat{\mathbf{r}}, 0)=\mathfrak{T}_{y}\left\{\mathfrak{T}_{x}^{-1}\left\{E_{x}(\hat{\mathbf{r}}, 0)\right\}\right\} .
\end{aligned}
$$

These imposed relations create the constraint on the electric fields that we deal with.

If the transforms $\mathfrak{T}_{\{x, y\}}$ are commutative with the free space propagation, that is,

$$
\mathfrak{T}_{\{x, y\}}\{S(\hat{\mathbf{r}}, 0)\} * * f_{z}(\hat{\mathbf{r}})=\mathfrak{T}_{\{x, y\}}\left\{S(\hat{\mathbf{r}}, 0) * * f_{z}(\hat{\mathbf{r}})\right\},
$$


for all $z$, then $E_{\{x, y\}}(\hat{\mathbf{r}}, z)$ can be computed from $S(\hat{\mathbf{r}}, z)$ at all $z$ planes by using the same transforms, $\mathfrak{T}_{\{x, y\}}$. Moreover, if $\mathfrak{T}_{\{x, y\}}$ commute with the free space propagation and the impulse responses given in Eqs. 8 and 18, then $E_{z}(\hat{\mathbf{r}}, z)$ and $\mathbf{H}(\hat{\mathbf{r}}, z)$ can be computed using $\mathfrak{T}_{\{x, y\}}$ and $S(\hat{\mathbf{r}}, z)$ at any $z$ plane as

$$
\begin{aligned}
& E_{z}(\hat{\mathbf{r}}, z)=\mathfrak{T}_{x}\left\{S(\hat{\mathbf{r}}, z) * * g_{x}(\hat{\mathbf{r}})\right\}+\mathfrak{T}_{y}\left\{S(\hat{\mathbf{r}}, z) * * g_{y}(\hat{\mathbf{r}})\right\}, \\
& H_{x}(\hat{\mathbf{r}}, z)=\mathfrak{T}_{x}\left\{S(\hat{\mathbf{r}}, z) * * g_{x x}(\hat{\mathbf{r}})\right\}+\mathfrak{T}_{y}\left\{S(\hat{\mathbf{r}}, z) * * g_{x y}(\hat{\mathbf{r}})\right\}, \\
& H_{y}(\hat{\mathbf{r}}, z)=\mathfrak{T}_{x}\left\{S(\hat{\mathbf{r}}, z) * * g_{y x}(\hat{\mathbf{r}})\right\}+\mathfrak{T}_{y}\left\{S(\hat{\mathbf{r}}, z) * * g_{y y}(\hat{\mathbf{r}})\right\}, \\
& H_{z}(\hat{\mathbf{r}}, z)=\mathfrak{T}_{x}\left\{\frac{j}{k \eta} \frac{\partial S(\hat{\mathbf{r}}, z)}{\partial y}\right\}-\mathfrak{T}_{y}\left\{\frac{j}{k \eta} \frac{\partial S(\hat{\mathbf{r}}, z)}{\partial x}\right\} .
\end{aligned}
$$

In this respect, $\mathfrak{T}_{\{x, y\}}$ can be used at an arbitrary $z$ plane in the computation of all components of the electromagnetic field and since $S(\hat{\mathbf{r}}, z)=S(\hat{\mathbf{r}}, 0) * * f_{z}(\hat{\mathbf{r}})$, specifying just $S(\hat{\mathbf{r}}, 0)$ is enough to find the electromagnetic field in $3 \mathrm{D}$ space. Moreover, if the scalar field, $S(\mathbf{r})$, is given over a curved surface in free space, the scalar field, and so the electromagnetic field, in 3D space can be found using the methods found in the literature that are developed for the scalar optical fields $[10,11,27,28]$.

Now, we choose $\mathfrak{T}_{\{x, y\}}$ as 2D LSI transforms so that Eqs. 23 and 24 are always satisfied. So, $\mathfrak{T}_{x}$ and $\mathfrak{T}_{y}$ can be associated by the impulse responses, $t_{x}(\hat{\mathbf{r}})$ and $t_{y}(\hat{\mathbf{r}})$, respectively, and the transforms can be written as a convolution operation; that is,

$E_{x}(\hat{\mathbf{r}}, 0)=S(\hat{\mathbf{r}}, 0) * * t_{x}(\hat{\mathbf{r}})$,

$E_{y}(\hat{\mathbf{r}}, 0)=S(\hat{\mathbf{r}}, 0) * * t_{y}(\hat{\mathbf{r}})$.

Therefore, since the convolution operation is commutative, if $\mathfrak{T}_{\{x, y\}}$ are 2D LSI transforms, then it is guaranteed that these transforms can be used to find all the components of the electric field in $3 \mathrm{D}$ space from a single scalar function $S(\hat{\mathbf{r}}, 0)$.

As stated in introduction part, there are some interpretations and works for the mapping of the scalar wave field to the electromagnetic wave field in free space in the literature. In [14], it is stated that a single scalar wave field may represent all the scalar components of electromagnetic vector wave field as each scalar component obeys the same wave equation; however, this is too restrictive. In [17], it is stated that the Kirchhoff diffraction formula for the scalar field is valid in the paraxial region by assuming that there is a single transverse component of the electric field; as we have already discussed, paraxial approximation is valid in too restrictive special cases, as well. Here in this section, it is shown that if $E_{x}(\hat{\mathbf{r}}, 0)$ and $E_{y}(\hat{\mathbf{r}}, 0)$ are related to each other through
$E_{x}(\hat{\mathbf{r}}, 0)=t_{x}(\hat{\mathbf{r}}) * *\left(t_{y}^{-1}(\hat{\mathbf{r}}) * * E_{y}(\hat{\mathbf{r}}, 0)\right)$

or equivalently

$E_{y}(\hat{\mathbf{r}}, 0)=t_{y}(\hat{\mathbf{r}}) * *\left(t_{x}^{-1}(\hat{\mathbf{r}}) * * E_{x}(\hat{\mathbf{r}}, 0)\right)$,

for some invertible $t_{\{x, y\}}(\hat{\mathbf{r}})$ where $t_{\{x, y\}}^{-1}(\hat{\mathbf{r}})$ represent the corresponding inverse system impulse responses, then a single scalar field characterizes the entire electromagnetic field. The constraints as expressed by Eqs. 26 and 27 extend the scalar wave representation of electromagnetic field propagation in free space to cover much wider situations beyond that is implied by [14] and [17].

In $[4,30]$, for a paraxial imaging setup in which the longitudinal components are assumed to be zero and by implicitly assuming that there is a relation such that $E_{y}(\mathbf{r}) / E_{x}(\mathbf{r})=$ $C \in \mathbb{C}$ for all $\mathbf{r}$, the scalar field is mapped to the vector field as

$E_{x, \text { con }}(\mathbf{r})=\frac{1}{\sqrt{1+|C|^{2}}} S(\mathbf{r})$
$E_{y, \text { con }}(\mathbf{r})=\frac{C}{\sqrt{1+|C|^{2}}} S(\mathbf{r})$,

where we refer the electric field components as a result of the conventional mapping as $E_{x, c o n}(\mathbf{r})$ and $E_{y, c o n}(\mathbf{r})$. Then, it is shown apart from a constant multiplier that

$|S(\mathbf{r})|^{2}=\left|\operatorname{Re}\left\{\mathbf{E}_{c o n}(\mathbf{r}) \times \mathbf{H}_{\text {con }}^{*}(\mathbf{r})\right\}\right|$,

which is the optical intensity [4]. For this case, again apart from a constant multiplier, it can also be shown that

$$
\begin{gathered}
|S(\mathbf{r})|^{2}=\left|\mathbf{E}_{\text {con }}(\mathbf{r})\right|^{2} \\
|\mathcal{S}(\hat{\mathbf{k}})|^{2}=\left|\mathcal{E}_{\text {con }}(\hat{\mathbf{k}})\right|^{2}
\end{gathered}
$$

and

$$
\begin{gathered}
|S(\mathbf{r})|^{2}=\left|\mathbf{H}_{\text {con }}(\mathbf{r})\right|^{2} \\
|\mathcal{S}(\hat{\mathbf{k}})|^{2}=\left|\mathcal{H}_{\text {con }}(\hat{\mathbf{k}})\right|^{2},
\end{gathered}
$$

where $\mathcal{S}(\hat{\mathbf{k}})=\mathfrak{F}_{2 D}\{S(\hat{\mathbf{r}}, 0)\}$. However, when the field is not paraxial, Eq. 30 cannot be satisfied due to the large magnitude of omitted $E_{z}(\mathbf{r})$ [18]. So, in the following section, we propose another scalar-to-vector wave field mapping by using some appropriate $t_{\{x, y\}}(\hat{\mathbf{r}})$ so that the power spectrum of the scalar field and the corresponding electric field and the magnetic field are also equal for wide-angle fields. 


\section{Inverse Filtering for Power Spectrum Equalization}

We define the power spectrum of an electric field as

$$
|\mathcal{E}(\hat{\mathbf{k}})|^{2}=\left|\mathcal{E}_{x}(\hat{\mathbf{k}})\right|^{2}+\left|\mathcal{E}_{y}(\hat{\mathbf{k}})\right|^{2}+\left|\mathcal{E}_{z}(\hat{\mathbf{k}})\right|^{2}
$$

which is also, apart from a constant multiplier, equal to the power spectrum of the magnetic field, $|\boldsymbol{H}(\hat{\mathbf{k}})|^{2}$. In terms of the assumed scalar field $\mathcal{S}(\hat{\mathbf{k}})$ and the transfer functions, $\mathcal{T}_{\{x, y\}}(\hat{\mathbf{k}})=\mathfrak{F}_{2 D}\left\{t_{\{x, y\}}(\hat{\mathbf{r}})\right\}$, Eq. 32 can be written as

$$
\begin{aligned}
|\mathcal{E}(\hat{\mathbf{k}})|^{2}= & \left|\mathcal{S}(\hat{\mathbf{k}}) \mathcal{T}_{x}(\hat{\mathbf{k}})\right|^{2}+\left|\mathcal{S}(\hat{\mathbf{k}}) \mathcal{T}_{y}(\hat{\mathbf{k}})\right|^{2} \\
& +\left|\mathcal{S}(\hat{\mathbf{k}})\left(\mathcal{T}_{x}(\hat{\mathbf{k}}) \mathcal{G}_{x}(\hat{\mathbf{k}})+\mathcal{T}_{y}(\hat{\mathbf{k}}) \mathcal{G}_{y}(\hat{\mathbf{k}})\right)\right|^{2} .
\end{aligned}
$$

Since our aim is to satisfy $|\mathcal{E}(\hat{\mathbf{k}})|^{2}=|\mathcal{S}(\hat{\mathbf{k}})|^{2}$, the required condition for this purpose becomes

$$
\begin{aligned}
1= & \left|\mathcal{T}_{x}(\hat{\mathbf{k}})\right|^{2}+\left|\mathcal{T}_{y}(\hat{\mathbf{k}})\right|^{2} \\
& +\left|\mathcal{T}_{x}(\hat{\mathbf{k}}) \mathcal{G}_{x}(\hat{\mathbf{k}})+\mathcal{T}_{y}(\hat{\mathbf{k}}) \mathcal{G}_{y}(\hat{\mathbf{k}})\right|^{2} .
\end{aligned}
$$

Therefore, if $\mathcal{T}_{\{x, y\}}(\hat{\mathbf{k}})$ are chosen such that Eq. 34 is satisfied, the power spectra of the scalar field and the corresponding electric and magnetic fields become equal. In this respect, when the magnitude square of the Fourier transform of an electric field can be recorded by a wide-angle imaging system, the proposed scalar-to-vector wave field mapping gives the exact results: The power spectrum of the scalar field and the power spectrum of the corresponding electric field match exactly. Or, it is known that an imaging system which uses a thin lens can record the intensity of the Fourier transform of the input scalar field under some paraxial approximations [14]. If the thin lens is not located along the axis of the imaging system and located at a tilted and rotated plane, the intensity image will be significantly incorrect when the conventional scalar-to-vector field mapping is used. However, no error will occur if the filters $\mathcal{T}_{\{x, y\}}(\hat{\mathbf{k}})$, satisfying Eq. 34, are used during the scalar-to-vector field mapping.

As an example, in addition to the equalized power spectrum, if the longitudinal component of the electric field is desired to be set to zero at each point in space, then $\mathcal{T}_{\{x, y\}}(\hat{\mathbf{k}})$ should be chosen as

$\mathcal{T}_{x}(\hat{\mathbf{k}})=\frac{k_{y} e^{j \theta(\hat{\mathbf{k}})}}{|\hat{\mathbf{k}}|}, \quad \mathcal{T}_{y}(\hat{\mathbf{k}})=-\frac{k_{x} e^{j \theta(\hat{\mathbf{k}})}}{|\hat{\mathbf{k}}|}$ where $\theta(\hat{\mathbf{k}})$ is an arbitrary phase function.

Please note that the power spectrum equalization method is used in the literature in image restoration algorithms $[1,6,15,16]$, where a blindly convolved and noise-added image is tried to be recovered from the measurements. Since $E_{z}(\mathbf{r})$ is computed using the filters $g_{\{x, y\}}(\hat{\mathbf{r}})$ from $E_{\{x, y\}}(\mathbf{r})$ using Eq. 7, $\mathcal{T}_{\{x, y\}}(\hat{\mathbf{k}})$ can be seen as the inverse filters which compensate the excessively large magnitudes of $\mathcal{G}_{\{x, y\}}(\hat{\mathbf{k}})$ that occur at large frequencies [18]. Since the measured quantity of the optical field can be the electric field intensity, that is defined as

$|\mathbf{E}(\mathbf{r})|^{2}=\left|E_{x}(\mathbf{r})\right|^{2}+\left|E_{y}(\mathbf{r})\right|^{2}+\left|E_{z}(\mathbf{r})\right|^{2}$,

by applying these inverse filters, it can be expected in $|\mathbf{E}(\mathbf{r})|^{2}$ that the excessively amplified large frequency components due to the $\mathcal{G}_{\{x, y\}}(\hat{\mathbf{k}})$ are compensated by $\mathcal{T}_{\{x, y\}}(\hat{\mathbf{k}})$.

After presenting the general constraints that the filters $\mathcal{T}_{\{x, y\}}(\hat{\mathbf{k}})$ should obey for equalized power spectra, now we specify the filters and proceed the analysis for simple polarization fields. That is $E_{y}(\mathbf{r}) / E_{x}(\mathbf{r})=C \in \mathbb{C}$ for all $\mathbf{r}$. If $C$ equals 1 , for example, the field is diagonally linearly polarized, or if $C=j$, the field is right-hand circularly polarized. As the special cases, $C$ can be zero or $\infty$, which correspond to $x$-polarized or $y$-polarized fields, respectively. The reason of this simple polarization restriction that we choose for the electric fields is not to create a difference between the power spectra of the $x$ and $y$ components of the electric fields. So, the relation between the scalar field and the electric field components become simple. Also, this polarization assumption is useful for many displays and spatial light modulators (SLM) that produce such electromagnetic fields with a simple polarization feature $[13,20,23,25,31,32]$. Please note that, although the assumption that we made on the polarization constraint is the same as the one made in the conventional scalar fields $[4,30]$, here we assume that the field that we deal with is a wide-angle field so that the longitudinal component cannot be neglected.

In the simply polarized fields, $\mathcal{T}_{y}(\hat{\mathbf{k}}) / \mathcal{T}_{x}(\hat{\mathbf{k}})$ becomes equal to $C$, as well. When $C \neq \infty$, using Eq. 34, $\left|\mathcal{T}_{x}(\hat{\mathbf{k}})\right|^{2}$ becomes

$$
\begin{aligned}
\left|\mathcal{T}_{x}(\hat{\mathbf{k}})\right|^{2} & = \begin{cases}\frac{1}{|C|^{2}+1+\left|\mathcal{G}_{x}(\hat{\mathbf{k}})+C \mathcal{G}_{y}(\hat{\mathbf{k}})\right|^{2}} & \text { if } k_{z} \neq 0 \\
0 & \text { if } k_{z}=0\end{cases} \\
& = \begin{cases}\frac{\left|k_{z}\right|^{2}}{\left|k_{z}\right|^{2}\left(|C|^{2}+1\right)+\left|k_{x}+C k_{y}\right|^{2}} & \text { if } k_{z} \neq 0 \\
0 & \text { if } k_{z}=0 .\end{cases}
\end{aligned}
$$


Therefore, $\mathcal{T}_{x}(\hat{\mathbf{k}})$ takes the form

$$
\begin{aligned}
\mathcal{T}_{x}(\hat{\mathbf{k}}) & = \begin{cases}\frac{e^{j \theta(\hat{\mathbf{k}})}}{\sqrt{|C|^{2}+1+\left|\mathcal{G}_{x}(\hat{\mathbf{k}})+C \mathcal{G}_{y}(\hat{\mathbf{k}})\right|^{2}}} & \text { if } k_{z} \neq 0 \\
0 & \text { if } k_{z}=0\end{cases} \\
& = \begin{cases}\frac{e^{j \theta(\hat{\mathbf{k}})\left|k_{z}\right|}}{\sqrt{\left|k_{z}\right|^{2}\left(|C|^{2}+1\right)+\left|k_{x}+C k_{y}\right|^{2}}} & \text { if } k_{z} \neq 0 \\
0 & \text { if } k_{z}=0 .\end{cases}
\end{aligned}
$$

Please note that different choices of the phase factor, $\theta(\hat{\mathbf{k}})$, do not violate Eq. 34. If, for example, the phases of the scalar field and the transverse components of the electric field are desired to be the same, then it can be chosen as zero. Or, if the phases of the scalar field and the $z$ component are desired to be the same, then it can be chosen as

$\theta(\hat{\mathbf{k}})=-\angle\left(\mathcal{G}_{x}(\hat{\mathbf{k}})+C \mathcal{G}_{y}(\hat{\mathbf{k}})\right)$.

Equating the phases of the scalar field and the $z$ component can be useful if the field consists only of plane waves whose propagation angles are large. In this case, as it will be shown below, since $\mathcal{T}_{x}(\hat{\mathbf{k}})$ is a low-pass filter, the dominant term in the electric field becomes the $z$ component. In this respect, the phase patterns of the scalar field and the dominant term of the electric field can be made equal by choosing $\theta(\hat{\mathbf{k}})$ as given by Eq. 39 .

When $C \neq \infty, \mathcal{T}_{y}(\hat{\mathbf{k}})$ can be found as $C \mathcal{T}_{x}(\hat{\mathbf{k}})$ using Eq. 38. If $C=\infty$, then $\mathcal{T}_{x}(\hat{\mathbf{k}})$ becomes zero and $\mathcal{T}_{y}(\hat{\mathbf{k}})$ can be computed as

$$
\begin{aligned}
\mathcal{T}_{y}(\hat{\mathbf{k}}) & = \begin{cases}\frac{e^{j \theta(\hat{\mathbf{k}})}}{\sqrt{1+\left|\mathcal{G}_{y}(\hat{\mathbf{k}})\right|^{2}}} & \text { if } k_{z} \neq 0 \\
0 & \text { if } k_{z}=0\end{cases} \\
& = \begin{cases}\frac{e^{j \theta(\hat{\mathbf{k}})\left|k_{z}\right|}}{\sqrt{k^{2}-k_{x}^{2}}} & \text { if } k_{z} \neq 0 \\
0 & \text { if } k_{z}=0 .\end{cases}
\end{aligned}
$$

In this case, $\theta(\hat{\mathbf{k}})$ can be chosen as

$\theta(\hat{\mathbf{k}})=-\angle \mathcal{G}_{y}(\hat{\mathbf{k}})$,

if the phases of the scalar field and the $z$ component are desired to be the same.

In Fig. 1, we show the magnitude responses of different $\mathcal{T}_{\{x, y\}}(\hat{\mathbf{k}})$ pairs based on Eqs. 38 and 40 for the propagating fields as grayscale images. From the figures, it can be said that all these transfer functions have a low-pass nature.
In the linearly polarized cases, as shown in Fig. 1a-c, the magnitude responses of the corresponding transfer functions are the rotated version of each other. So, it can be shown that if the electric field has a different linear polarization, i.e., $C \in \mathbb{R}$, then the corresponding transfer functions will be another rotated version where the amount of rotation is determined by $C$. Also, in the circularly polarized case, the filter magnitude responses show a circularly symmetric pattern, so it can be said that the orientation characteristics of the scalar wave field are preserved in the resulting $x$ and $y$ components of the electric field.

At the end of the scalar-to-vector wave field mapping, the resulting electric field components in the Fourier domain become

$$
\begin{aligned}
& \mathcal{E}_{x}(\hat{\mathbf{k}})= \begin{cases}\mathcal{S}(\hat{\mathbf{k}}) \mathcal{T}_{x}(\hat{\mathbf{k}}) & \text { if } C \neq \infty \\
0 & \text { if } C=\infty,\end{cases} \\
& \mathcal{E}_{y}(\hat{\mathbf{k}})= \begin{cases}C \mathcal{S}(\hat{\mathbf{k}}) \mathcal{T}_{x}(\hat{\mathbf{k}}) & \text { if } C \neq \infty \\
\mathcal{S}(\hat{\mathbf{k}}) \mathcal{T}_{y}(\hat{\mathbf{k}}) & \text { if } C=\infty,\end{cases} \\
& \mathcal{E}_{z}(\hat{\mathbf{k}})= \begin{cases}\mathcal{S}(\hat{\mathbf{k}}) \mathcal{T}_{x}(\hat{\mathbf{k}})\left(\mathcal{G}_{x}(\hat{\mathbf{k}})+C \mathcal{G}_{y}(\hat{\mathbf{k}})\right) & \text { if } C \neq \infty \\
\mathcal{S}(\hat{\mathbf{k}}) \mathcal{T}_{y}(\hat{\mathbf{k}}) \mathcal{G}_{y}(\hat{\mathbf{k}}) & \text { if } C=\infty .\end{cases}
\end{aligned}
$$

As a final note to this section, a direct consequence of equalizing the power spectra of the scalar and vector fields in the space domain can be written as

$$
\begin{aligned}
S(\hat{\mathbf{r}}, z) * * S^{*}(-\hat{\mathbf{r}}, z)= & E_{x}(\hat{\mathbf{r}}, z) * * E_{x}^{*}(-\hat{\mathbf{r}}, z) \\
& +E_{y}(\hat{\mathbf{r}}, z) * * E_{y}^{*}(-\hat{\mathbf{r}}, z) \\
& +E_{z}(\hat{\mathbf{r}}, z) * * E_{z}^{*}(-\hat{\mathbf{r}}, z)
\end{aligned}
$$

One of the results that can be obtained from Eq. 43 is

$\iint_{-\infty}^{\infty}|\mathbf{E}(\hat{\mathbf{r}}, z)|^{2} \mathrm{~d} \hat{\mathbf{r}}=\int_{-\infty}^{\infty}|S(\hat{\mathbf{r}}, z)|^{2} \mathrm{~d} \hat{\mathbf{r}}$,

for all $z$. Equation 44 states that the total powers of the scalar field and the corresponding electric field are always equal at all $z$ planes. Therefore, it can be said that the spatially average difference between $|\mathbf{E}(\hat{\mathbf{r}}, z)|^{2}$ and $|S(\hat{\mathbf{r}}, z)|^{2}$ is always zero.

\section{Simulation Results}

In this section, we present some discrete simulation results in order to compare the performances of the conventional and proposed scalar-to-vector wave field mapping on the reconstruction of the corresponding electric field intensity pattern at $z=0$ plane. That is, we assume that a scalar wave field 


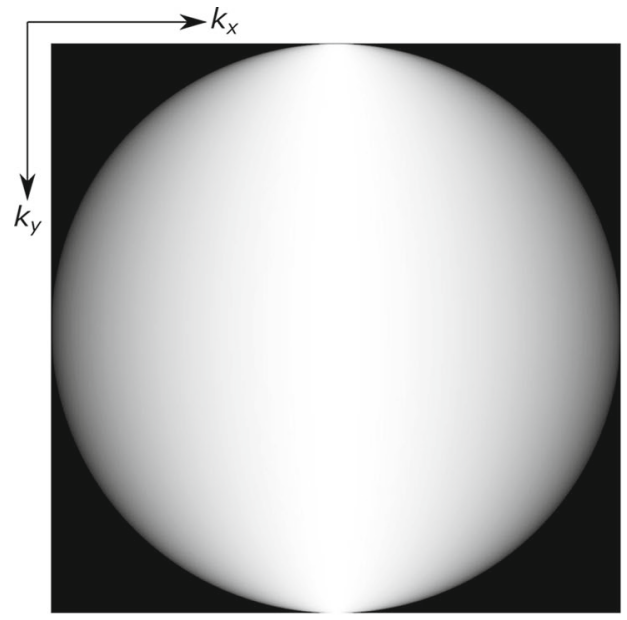

(a)

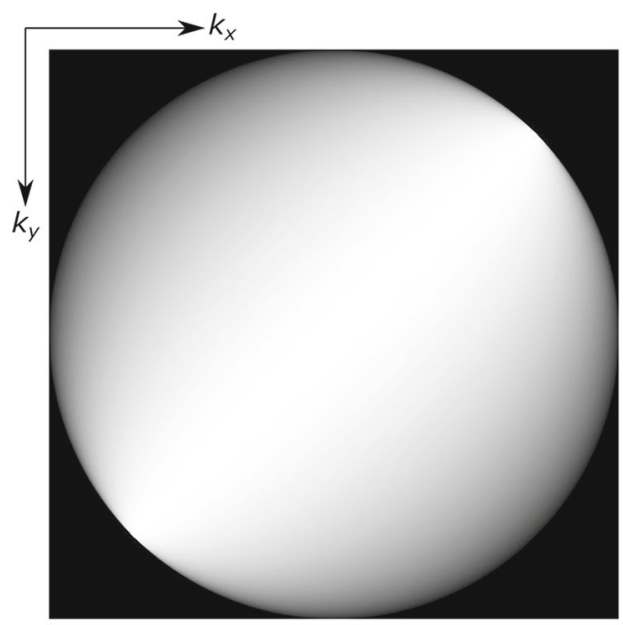

(c)

Fig. 1 Magnitudes of the corresponding transfer functions which satisfy Eq. 34 are shown for different polarization propagating cases. The centers of the images correspond to $\left(k_{x}, k_{y}\right)=(0,0)$. a The magnitude of $\mathcal{T}_{x}(\hat{\mathbf{k}})$ is shown as the result of Eq. 38 for the propagating fields when the field is $x$-polarized, i.e., $C=0$. b The magnitude of $\mathcal{T}_{y}(\hat{\mathbf{k}})$ is shown as the result of Eq. 40 for the propagating fields when the field

in space domain is given and the magnitude square of that field is desired to be generated as the electric field intensity pattern. In the conventional scalar case, the scalar wave field is mapped to $E_{\{x, y\}}(\hat{\mathbf{r}}, 0)$ according to Eq. 28. For the proposed scalar case, on the other hand, $E_{\{x, y\}}(\hat{\mathbf{r}}, 0)$ are computed using Eq. 42 from the given scalar field and the transfer functions, $\mathcal{T}_{\{x, y\}}(\hat{\mathbf{k}})$, that are given by Eqs. 38 and 40 . In both the conventional and proposed scalar fields, $E_{z}(\hat{\mathbf{r}}, 0)$ is computed using the $x$ and $y$ components of the electric field and the transfer functions, $\mathcal{G}_{\{x, y\}}(\hat{\mathbf{k}})$, as given by Eq. 9 .

Here, we first summarize the steps of the conversion from continuous domain to discrete domain. The details of this procedure can be found in [18]. Since we assume that there

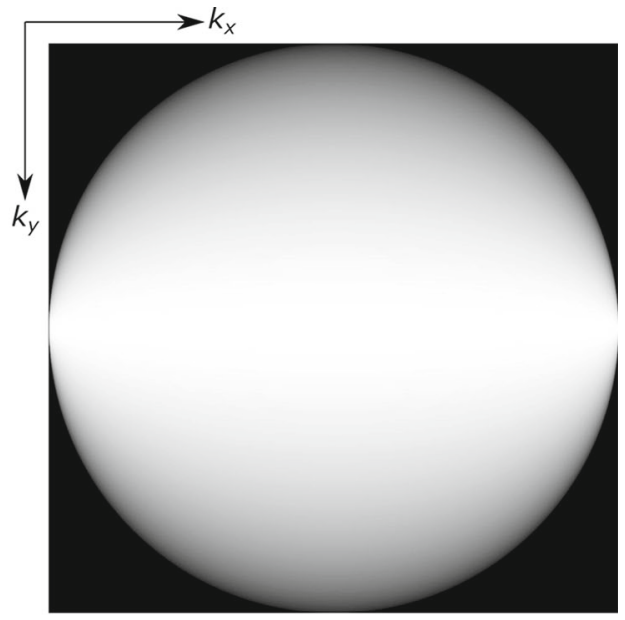

(b)

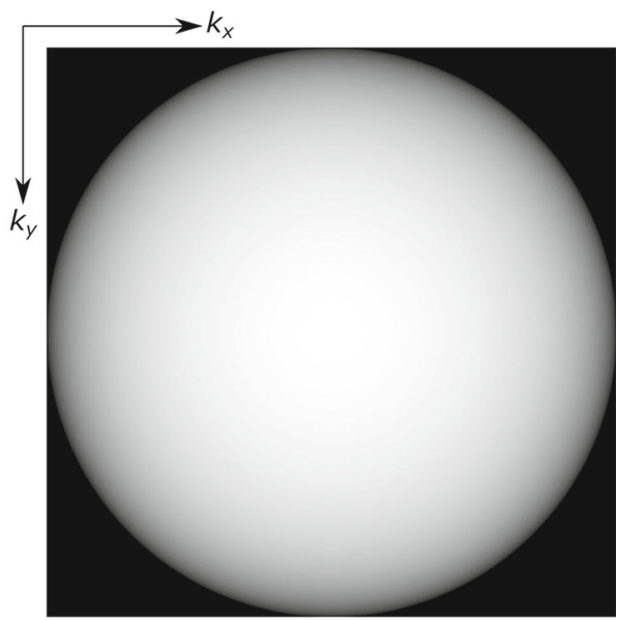

(d)

is $y$-polarized, i.e., $C=\infty$. c The magnitude of $\mathcal{T}_{x}(\hat{\mathbf{k}})=\mathcal{T}_{y}(\hat{\mathbf{k}})$ is shown as the result of Eq. 38 for the propagating fields when the field is diagonally linearly polarized, i.e., $C=1$. $\mathbf{d}$ The magnitude of $\mathcal{T}_{x}(\hat{\mathbf{k}})= \pm j \mathcal{T}_{y}(\hat{\mathbf{k}})$ is shown as the result of Eq. 38 for the propagating fields when the field is circularly polarized, i.e., $C= \pm j$

is no plane wave components when $|\hat{\mathbf{k}}| \geq k$ in the electromagnetic wave field that we deal with, by choosing the sampling period along both $x$ and $y$ directions, which are $X_{S}$ and $Y_{s}$, respectively, greater than $\pi / k$, one can satisfy the Shannon-Nyquist sampling theorem [24]. However, since our aim is to present results which mimic continuous fields, for better illustration, we choose different sampling periods for different simulations.

We assume that the field components take the form $R\left(n X_{s}, m Y_{s}, 0\right) \equiv \tilde{R}[n, m]$, where $n$ and $m$ are integers. Please note that, since all the computations are done for $z=0$ plane, we omit this coordinate axis in our notation for the discrete fields. Here $R(\hat{\mathbf{r}}, 0)$ may represent $S(\hat{\mathbf{r}}, 0)$ or one of the components of $\mathbf{E}(\hat{\mathbf{r}}, 0)$. Please note that we assume 
$\tilde{R}[n, m]$ is periodic by $N \in \mathbb{Z}^{+}$and $M \in \mathbb{Z}^{+}$along $n$ and $m$ directions, respectively.

We do the field computations in the frequency domain. For this purpose, the 2D and size $N \times M$ discrete Fourier transform (DFT) and its inverse (IDFT) for $p \in[0, N-1]$ and $q \in[0, M-1]$ are defined as,

$\tilde{\mathcal{R}}[p, q]=\sum_{n=0}^{N-1} \sum_{m=0}^{M-1} \tilde{R}[n, m] e^{-j 2 \pi\left(\frac{p n}{N}+\frac{q m}{M}\right)}$,

$\tilde{R}[n, m]=\frac{1}{N M} \sum_{p=0}^{N-1} \sum_{q=0}^{M-1} \tilde{\mathcal{R}}[p, q] e^{j 2 \pi\left(\frac{p n}{N}+\frac{q m}{M}\right)}$,

respectively [24]. We denote the 2D DFT and IDFT operations as $\tilde{\mathcal{R}}[p, q]=\mathcal{D}_{N \times M}\{\tilde{R}[n, m]\}$ and $\tilde{R}[n, m]=$ $\mathcal{D}_{N \times M}^{-1}\{\tilde{\mathcal{R}}[p, q]\}$.

The transfer functions of the filters in the discrete domain are obtained by periodically replicating and sampling the transfer functions of the filters in the continuous domain. The final version of the discrete filters takes the form

$\left.\tilde{\mathcal{B}}[p, q]\right|_{\substack{p=\hat{p}_{\bmod N} \\ q=\hat{q}_{\bmod M}}}=\mathcal{B}\left(\frac{2 \pi}{N X_{S}} \hat{p}, \frac{2 \pi}{M Y_{S}} \hat{q}\right)$,

where $\hat{p} \in\left[-\left\lceil\frac{N-1}{2}\right\rceil,\left\lceil\frac{N}{2}-1\right\rceil\right], \hat{q} \in\left[-\left\lceil\frac{M-1}{2}\right\rceil\right.$, $\left.\left\lceil\frac{M}{2}-1\right\rceil\right],\lceil\cdot\rceil$ is the ceiling operator which rounds a decimal number to the nearest larger integer. Here $\mathcal{B}(\hat{\mathbf{k}})$ may represent $\mathcal{G}_{\{x, y\}}(\hat{\mathbf{k}})$ or $\mathcal{T}_{\{x, y\}}(\hat{\mathbf{k}})$.

In the simulations, we begin with a given scalar field $\tilde{S}[n, m]$ and the magnitude square of that scalar field, $\tilde{P}[n, m]=|\tilde{S}[n, m]|^{2}$, as the desired electric field intensity pattern. Then, in the conventional scalar mapping, the electric field components are found in the DFT domain as

$\tilde{\mathcal{E}}_{x, \operatorname{con}}[p, q]=\frac{1}{\sqrt{1+|C|^{2}}} \tilde{\mathcal{S}}[p, q]$,

$\tilde{\mathcal{E}}_{y, \text { con }}[p, q]=\frac{C}{\sqrt{1+|C|^{2}}} \tilde{\mathcal{S}}[p, q]$,

where $\tilde{\mathcal{S}}[p, q]=\mathcal{D}_{N \times M}\{\tilde{S}[n, m]\}$. For the proposed scalar mapping, the field components are found as

$$
\begin{aligned}
& \tilde{\mathcal{E}}_{x, p r o}[p, q]= \begin{cases}\tilde{\mathcal{T}}_{x}[p, q] \tilde{\mathcal{S}}[p, q] & \text { if } C \neq \infty \\
0 & \text { otherwise, }\end{cases} \\
& \tilde{\mathcal{E}}_{y, p r o}[p, q]= \begin{cases}C \tilde{\mathcal{T}}_{x}[p, q] \tilde{\mathcal{S}}[p, q] & \text { if } C \neq \infty \\
\tilde{\mathcal{T}}_{y}[p, q] \tilde{\mathcal{S}}[p, q] & \text { otherwise, }\end{cases}
\end{aligned}
$$

where $\tilde{\mathcal{T}}_{\{x, y\}}[p, q]$ are computed from $\mathcal{T}_{\{x, y\}}(\hat{\mathbf{k}})$ by using Eq. 46. Please note that $\mathcal{T}_{x}(\hat{\mathbf{k}})$ and $\mathcal{T}_{y}(\hat{\mathbf{k}})$ are given by Eqs. 38 and 40, respectively. Finally, the corresponding $z$ components are computed in the DFT domain as

$\tilde{\mathcal{E}}_{z, \text { con }}[p, q]=\tilde{\mathcal{G}}_{x}[p, q] \tilde{\mathcal{E}}_{x, \text { con }}[p, q]+\tilde{\mathcal{G}}_{y}[p, q] \tilde{\mathcal{E}}_{y, \text { con }}[p, q]$,

$\tilde{\mathcal{E}}_{z, p r o}[p, q]=\tilde{\mathcal{G}}_{x}[p, q] \tilde{\mathcal{E}}_{x, p r o}[p, q]+\tilde{\mathcal{G}}_{y}[p, q] \tilde{\mathcal{E}}_{y, p r o}[p, q]$,

where $\tilde{\mathcal{G}}_{\{x, y\}}[p, q]$ are again computed from $\mathcal{G}_{\{x, y\}}(\hat{\mathbf{k}})$ by using Eq. 46. Finally, the discrete intensities generated as a result of the conventional and proposed scalar mappings are computed as

$$
\begin{aligned}
& \tilde{P}_{\text {con }[n, m]}=\left|\mathcal{D}_{N \times M}^{-1}\left\{\tilde{\mathcal{E}}_{\text {con }}[p, q]\right\}\right|^{2}, \\
& \tilde{P}_{\text {pro }}[n, m]=\left|\mathcal{D}_{N \times M}^{-1}\left\{\tilde{\mathcal{E}}_{\text {pro }}[p, q]\right\}\right|^{2},
\end{aligned}
$$

where $\tilde{\mathcal{E}}_{\text {con }}[p, q]=\left[\tilde{\mathcal{E}}_{x, \text { con }}[p, q] \tilde{\mathcal{E}}_{y, \text { con }}[p, q] \tilde{\mathcal{E}}_{z, \text { con }}\right.$ $[p, q]]^{T}$ and $\tilde{\mathcal{E}}_{\text {pro }}[p, q]=\left[\tilde{\mathcal{E}}_{x, p r o}[p, q] \tilde{\mathcal{E}}_{y, \operatorname{pro}}[p, q]\right.$ $\left.\tilde{\mathcal{E}}_{z, \text { pro }}[p, q]\right]^{T}$. The 2D IDFT operation in Eq. 50 is applied to each component of $\tilde{\mathcal{E}}_{\text {con }}[p, q]$ and $\tilde{\mathcal{E}}_{\text {pro }}[p, q]$, separately.

Finally, we compare three intensity patterns. These are the desired intensity pattern $\tilde{P}[n, m]$, the pattern generated as the result of conventional scalar-to-vector field mapping, $\tilde{P}_{c o n}[n, m]$, and the pattern generated as the result of proposed scalar-to-vector field mapping, $\tilde{P}_{\text {pro }}[n, m]$.

In order to test the effect of the inverse filtering approach, in the first simulation, we choose $X_{s}=Y_{s}=\lambda / 2$, which is the Nyquist rate for the propagating fields, and a 2D chirp signal as the scalar field for $N=M=1024$ as,

$$
\begin{aligned}
& \tilde{S}[n, m]= \\
& \mathcal{D}_{N \times M}^{-1}\left\{\mathcal{D}_{N \times M}\left\{\cos \left(\frac{\pi}{N}\left[\left(n-\frac{N}{2}\right)^{2}+\left(m-\frac{M}{2}\right)^{2}\right]\right)\right\}\right. \\
& Q[p, q]\}
\end{aligned}
$$

where $Q[p, q]$ matrix,

$$
\left.Q[p, q]\right|_{\substack{p=\hat{p}_{\bmod N} \\ q=\hat{q}_{\bmod M}}}= \begin{cases}1 & \text { if } \hat{p}^{2} / N^{2}+\hat{q}^{2} / M^{2}<1 / 4 \\ 0 & \text { otherwise. }\end{cases}
$$

is introduced to remove the evanescent components. Please note that we choose this test pattern, because its instantaneous normalized frequency includes the full frequency range $[-\pi, \pi)$ with equal strength. Hence, we are able to 


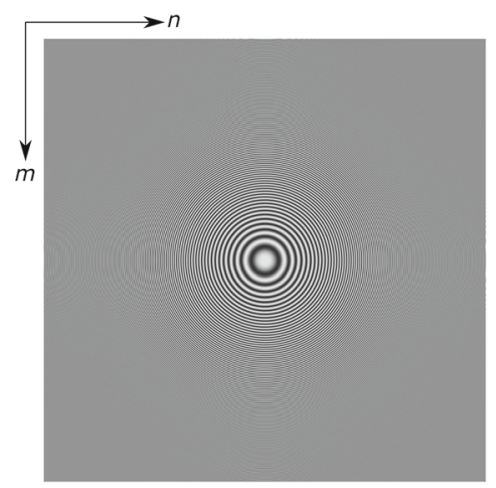

(a)

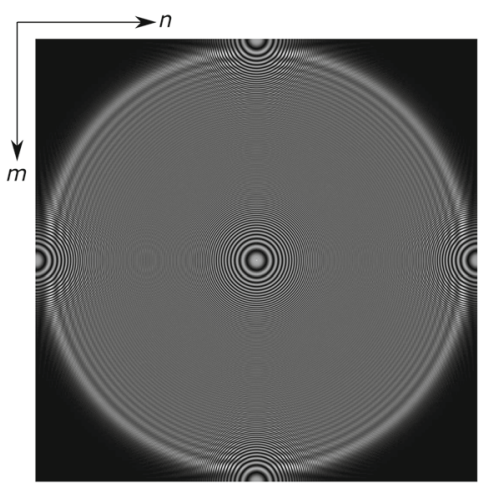

(b)

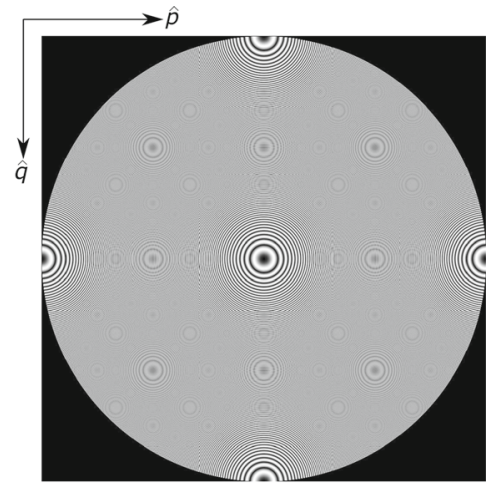

(c)
Fig. 2 The test patterns which are given by Eqs. 51 and 53 are shown. $n$ and $\hat{p}$ increase as going from left to right and $m$ and $\hat{q}$ increase as going from top to bottom. The top left of the images shown in $(\mathbf{a}, \mathbf{b})$

test the success of the inverse filter in the reconstruction of all the possible frequency components. The magnitude of the 2D DFT of this field is [22]

$|\tilde{\mathcal{S}}[p, q]|=N\left|\sin \left(\frac{\pi}{N}\left(p^{2}+q^{2}\right)\right)\right| Q[p, q]$.

In Fig. 2, these patterns given by Eqs. 51 and 53 as well as $\tilde{P}[n, m]=|\tilde{S}[n, m]|^{2}$ are shown. Please note that the shifted version of the magnitude of the 2D DFT of the test pattern is shown in Fig. 2c. As a consequence of the shift, the centers of the images correspond to zero frequency and the normalized frequency range extends from $-N / 2$ to $N / 2-1$.

The reconstructed intensity patterns for the first simulation are shown in Figs. 3 and 4 for $C=1$ and $C=-j$, respectively. Although it is desirable to show $\tilde{P}[n, m], \tilde{P}_{c o n}[n, m]$ and $\tilde{P}_{\text {pro }}[n, m]$ using the same grayscale range for comparison, $\tilde{P}[n, m]$ and $\tilde{P}_{\text {pro }}[n, m]$ become invisible due to the dominant high-frequency regions of $\tilde{P}_{c o n}[n, m]$ when the same grayscale is used; in other words, the conventional method gives a result that is grossly incorrect. So, for the illustration purposes, before jointly scaling the results in gray levels, we linearly shrank the contrast of the grayscale image to get $\hat{P}_{c o n}[n, m]$ from $\tilde{P}_{c o n}[n, m] . \tilde{P}[n, m], \hat{P}_{c o n}[n, m]$, $\tilde{P}_{\text {pro }}[n, m]$ are shown in Fig. $3 \mathrm{a}-\mathrm{c}$ for $C=1$ and in Fig. $4 \mathrm{a}-$ c for $C=-j$. Since it is more informative, we also provide the reconstructed patterns for the conventional case in logarithmic scale in Fig. 3e and 4e. From these figures, it can be said that the intensity pattern that is desired to be reconstructed is completely lost if the conventional procedure is used. Also, the value of $C$ affects the resulting conventional mapping intensity patterns. In the diagonally linearly polarized case, as shown in Fig. 3b, e, the reconstructed intensity pattern due to the conventional mapping shows an corresponds to $(n, m)=(0,0)$, and the center of the image shown in (c) corresponds to $(\hat{p}, \hat{q})=(0,0)$. a $\tilde{S}[n, m]$. b $\tilde{P}[n, m]$. c Shifted $|\tilde{\mathcal{S}}[p, q]|$

unequal distribution in high-frequency regions. Such a result occurs due to the constructive and destructive superposition of $\tilde{\mathcal{G}}_{x}[p, q]$ and $\tilde{\mathcal{G}}_{y}[p, q]$ in the high-frequency regions. In the left-hand circularly polarized case, as shown in Fig. 4b, e these constructive and destructive superpositions end up with a circularly symmetric distribution. On the other hand, due to the proposed mapping, the reconstructed intensity patterns, as shown in Figs. 3c and 4c for the jointly scaled cases and in Figs. 3d and $4 d$ for the independently scaled cases, preserve the original intensity pattern. Therefore, the inverse filters $\tilde{\mathcal{T}}_{\{x, y\}}[p, q]$ again compensate the high-pass effect of $\tilde{\mathcal{G}}_{\{x, y\}}[p, q]$ for this simulation, as well. Also, for the proposed mapping, there is not much difference between the reconstructed intensity patterns for $C=j$ and $C=1$ cases. Therefore, it can be said that different values of $C$ do not affect the resulting intensity pattern and the effect of $C$ on the reconstructed intensity pattern disappears, thanks to the inverse filters, in these simulations.

In the second simulation, we test the proposed algorithm for a realistic intensity pattern, as shown in Fig. 5a. We assume that the scalar field that generates this intensity pattern is zero phase; so, the scalar field can be found by computing the square root of the given intensity pattern. In this simulation, there are two main differences from the previous simulation. The first one is the sampling rate, which is used to convert the analog signals to the discrete signals. Although $X_{s}=Y_{s}=\lambda / 2$ was enough to show the deteriorations due to the conventional mapping in the previous simulation, in this example this sampling rate is not enough to simulate the actual analog operations and show meaningful results. Moreover, since the rate of change of $\mathcal{G}_{\{x, y\}}(\hat{\mathbf{k}})$ is large at around $|\hat{\mathbf{k}}|=k[18]$, the simulation results are highly dependent on the chosen numerical parameters. In this simulation, we choose $X_{s}=Y_{s}=\lambda / 4.75$ as the sam- 


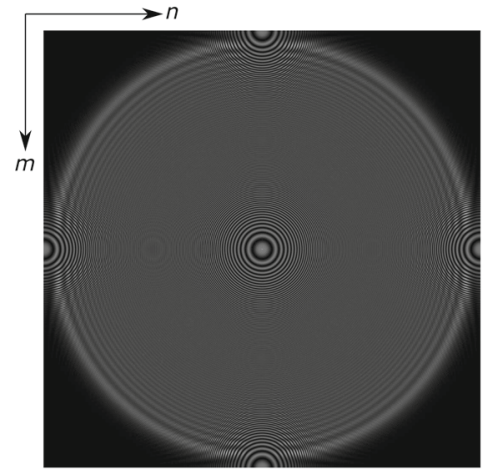

(a)

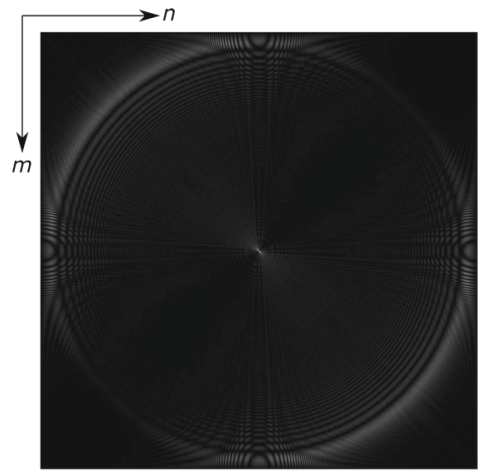

(b)

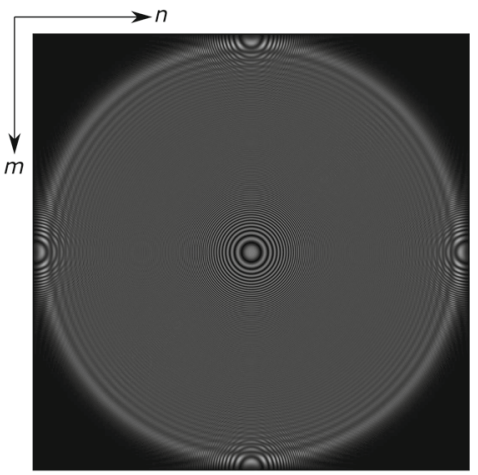

(c)

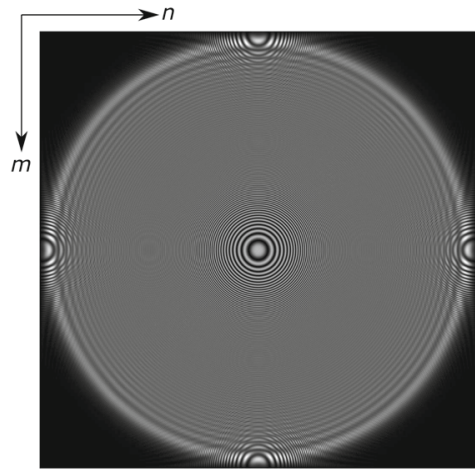

(d)

Fig. 3 Reconstructed intensity patterns for the first simulation are shown for $C=1$, i.e., diagonally polarized field. The original intensity pattern is shown in (a). The resulting intensity patterns due to the conventional and proposed mappings are shown in (b), and (c), respectively. Before jointly mapping the patterns to the grayscale range, the contrast

pling rate. As will be shown below, at this sampling rate, the actual continuous field will be simulated according to the expected behavior of the filters and the deterioration due to the conventional mapping will be severe. The second difference in this simulation is that the chosen intensity pattern is assumed to be generated using a holographic reconstruction technique. For this purpose, we assume that a SLM which is capable of producing a full complex light field is located at $z=0$ plane. Then, the given intensity pattern is desired to be generated by the optical field propagated from this SLM to the image plane located at $z=20 \mathrm{~cm}$ away from the SLM plane. Both the size of the intensity pattern and the dimensions of the discrete SLM are taken in this example as $1566 \times 560$. Please note that, with this sampling rate and the size of the intensity patterns, the actual dimension of the SLM becomes on the order of $100 \mu \mathrm{m}$, that is quite small for a realistic application. However, here our aim is to simulate the proposed and conventional mappings over a familiar intensity pattern, which can be encountered frequently in nature, rather than making a computer simulation

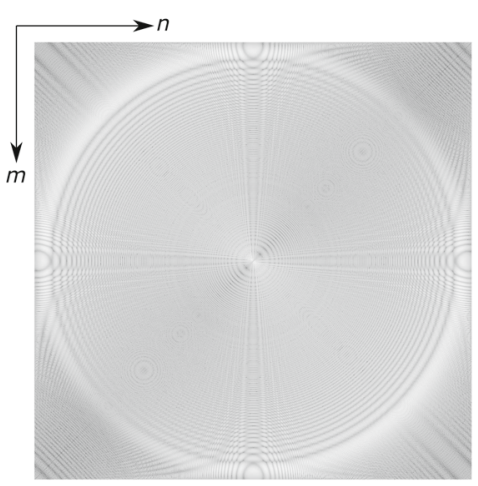

(e)

of $\tilde{P}_{c o n}[n, m]$ is linearly shrank to get $\hat{P}_{c o n}[n, m]$. d Shows the independently scaled version of (c). e Shows $\hat{P}_{c o n}[n, m]$ in the log scale. The top left corner of the images corresponds to $(n, m)=(0,0)$. a $\tilde{P}[n, m]$. b $\hat{P}_{\text {con }}[n, m]$. c $\tilde{P}_{\text {pro }}[n, m]$. d Independently scaled $\tilde{P}_{\text {pro }}[n, m]$. e $\ln \left(\hat{P}_{\text {con }}[n, m]\right)$

of a realistic optical setup. We do the simulation for a single polarization case such that the SLM does not produce the $y$ component, i.e., $C=0$. Moreover, in order to simulate the free space propagation, we use Rayleigh-Sommerfeld diffraction formula in the 2D DFT domain [7,9] with the wavelength $\lambda=500 \mathrm{~nm}$. As a result, in the conventional mapping, the initial complex-valued scalar field is written onto the SLM without making a modification and in the proposed mapping, that scalar field is written onto the SLM after applying the inverse filter.

In Fig. 5b, c, the magnitudes of the written complex-valued scalar fields onto the SLM are shown for the conventional and proposed mappings, respectively, where it is difficult to observe a difference. However, the resulting intensity patterns, reconstructed at the image plane, show a significant difference as shown in Fig. 6. In Fig. 6a-c, the original intensity pattern and the resulting intensity patterns due to the conventional and proposed mappings are shown, respectively. Please note that these three patterns are mapped to the grayscale images using the same scale. Moreover, in Fig. 6d, 


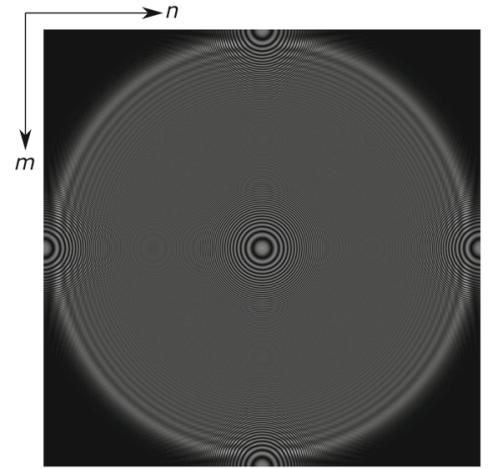

(a)

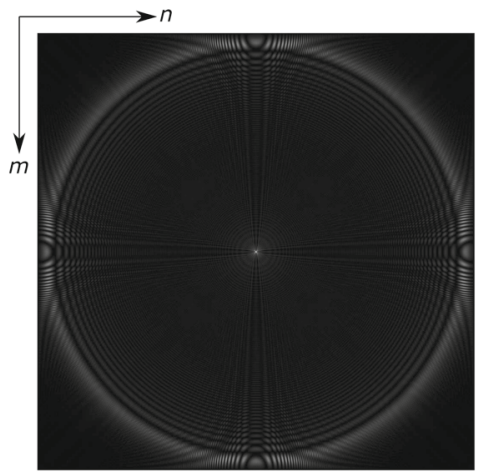

(b)

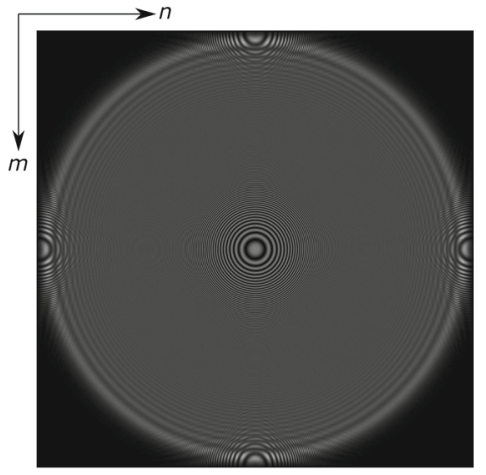

(c)

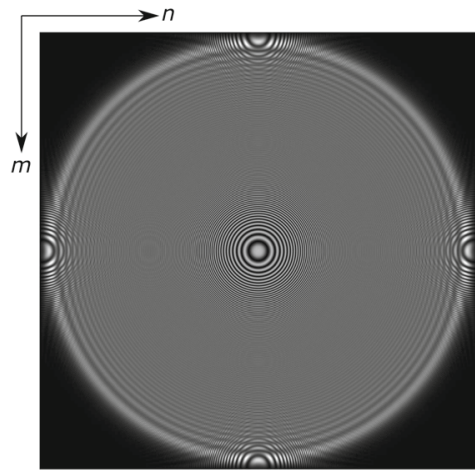

(d)

Fig. 4 Reconstructed intensity patterns for the first simulation are shown for $C=-j$, i.e., left-hand circularly polarized field. The original intensity pattern is shown in (a). The resulting intensity patterns due to the conventional and proposed mappings are shown in (b) and (c), respectively. Before jointly mapping the patterns to the

the resulting intensity pattern due to the proposed mapping is shown in full grayscale range. Please note that, since the computations are performed in the DFT domain, the shown patterns represent one period of their periodically replicated versions. In the conventional case, the artifacts due to the amplified frequencies can be seen clearly. Due to the branches of the tree and the edges of the buildings, a variation along the horizontal direction, and hence, nonzero frequency components for $\left|k_{x}\right| \approx k$ occur. As a result of these high frequencies, some periodic patterns from left to right emerge in the conventional case due to the excessive amplification, as shown in Figure $6 \mathrm{~b}$. However, these artifacts disappear in the intensity patterns when the proposed scalar mapping method is used. Although there are still some minor differences between the original pattern and the intensity pattern obtained by the proposed method, they look quite similar and the artifacts due to the conventional mapping are clearly eliminated. Therefore, it can be said that the inverse filters compensate the deteriorations which occur when the conventional methods are used.

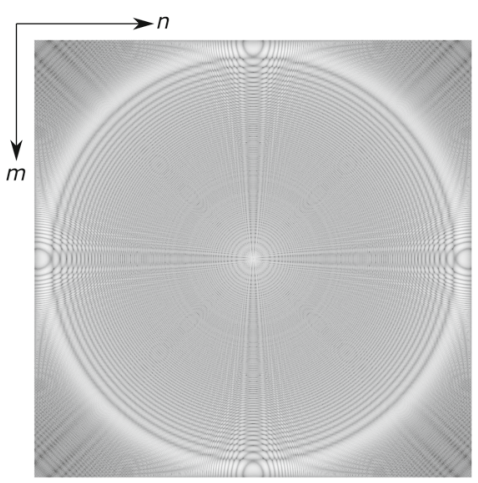

(e)

grayscale range, the contrast of $\tilde{P}_{c o n}[n, m]$ is linearly shrank to get $\hat{P}_{c o n}[n, m]$. d Shows the independently scaled version of (c). e Shows $\hat{P}_{c o n}[n, m]$ in the log scale. The top left corner of the images corresponds to $(n, m)=(0,0)$. a $\tilde{P}[n, m]$. b $\hat{P}_{c o n}[n, m]$. c $\tilde{P}_{p r o}[n, m]$. d Independently scaled $\tilde{P}_{\text {pro }}[n, m]$. e $\ln \left(\hat{P}_{\text {con }}[n, m]\right)$

\section{Conclusions}

In this paper, a novel scalar-to-vector wave field mapping for monochromatic electromagnetic wave fields is developed. Under the proposed mapping, the scalar wave field obeys the wave propagation rules. It is presented that the power spectra of the scalar field and the corresponding electric field become equal if the transverse components of the electric field are generated from the scalar field as

$$
\begin{aligned}
& \mathcal{E}_{x}(\hat{\mathbf{k}})=\mathcal{S}(\hat{\mathbf{k}}) \mathcal{T}_{x}(\hat{\mathbf{k}}) \\
& \mathcal{E}_{y}(\hat{\mathbf{k}})=\mathcal{S}(\hat{\mathbf{k}}) \mathcal{T}_{y}(\hat{\mathbf{k}}),
\end{aligned}
$$

where the linear-shift invariant filters $\mathcal{T}_{\{x, y\}}(\hat{\mathbf{k}})$ satisfy

$$
\begin{aligned}
1= & \left|\mathcal{T}_{x}(\hat{\mathbf{k}})\right|^{2}+\left|\mathcal{T}_{y}(\hat{\mathbf{k}})\right|^{2}+\mid \mathcal{T}_{x}(\hat{\mathbf{k}}) \mathcal{G}_{x}(\hat{\mathbf{k}}) \\
& +\left.\mathcal{T}_{y}(\hat{\mathbf{k}}) \mathcal{G}_{y}(\hat{\mathbf{k}})\right|^{2} .
\end{aligned}
$$




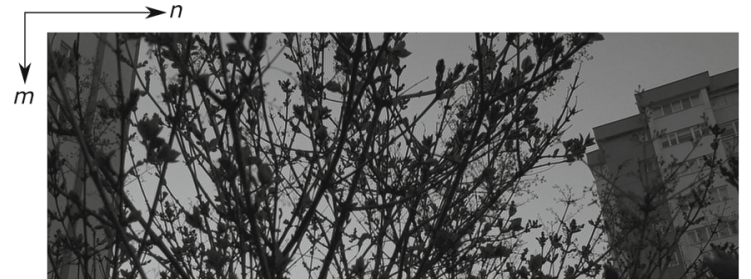

(a)

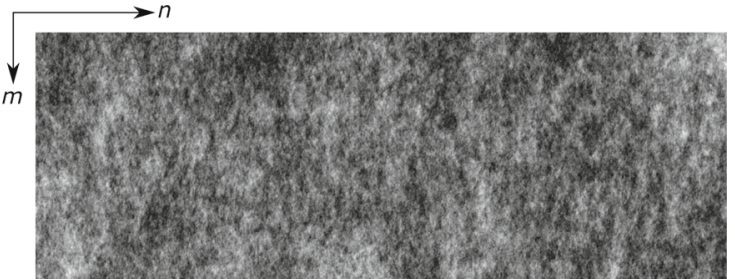

(b)

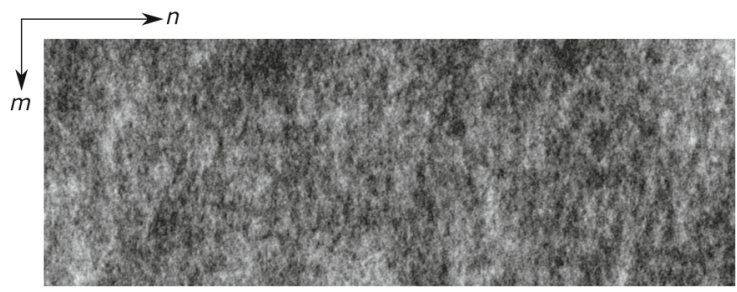

(c)

Fig. 5 The test patterns for the second simulation are shown. The image plane is assumed to be located $20 \mathrm{~cm}$ away from the SLM. Wavelength is $500 \mathrm{~nm}$. The top left corner of the images corresponds to $(n, m)=(0,0)$. a The intensity pattern that is desired to be holograph- ically generated by the SLM at the image plane. b The magnitude of the complex-valued scalar field that is written onto the SLM (conventional case). $\mathbf{c}$ The magnitude of the complex-valued scalar field written onto the SLM (proposed case)

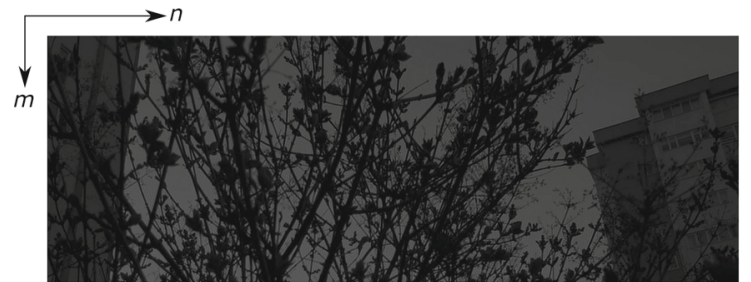

(a)

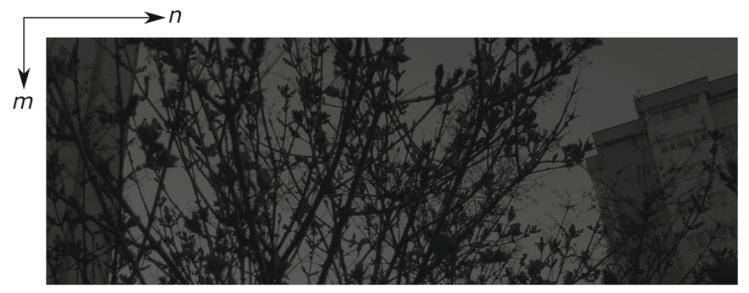

(c)

Fig. 6 Reconstructed intensity patterns are shown for $C=0$, i.e., $x$ polarized field. The original intensity pattern shown in Fig. 5a, and the intensity patterns as the result of the conventional and proposed mappings are shown in (a, b, and $\mathbf{c})$, respectively. The patterns shown in

As a result of the power spectrum equalization, total powers of the scalar field and the corresponding electric field are always equal at any arbitrary $z$ plane. The filters are also specified for electric fields with either zero longitudinal component or simple polarization features. The resulting filters for simple polarization cases compensate the excessive amplification of $\mathcal{G}_{\{x, y\}}(\hat{\mathbf{k}})$ when $|\hat{\mathbf{k}}| \approx k$.

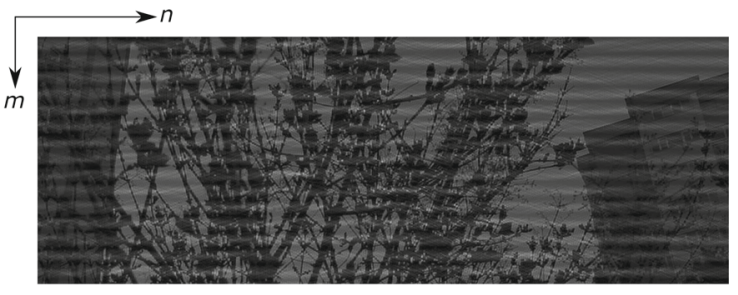

(b)

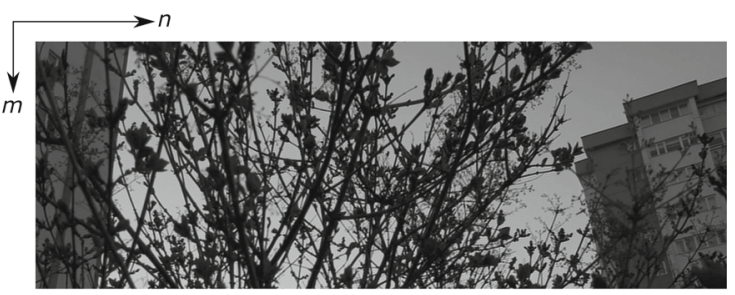

(d)

these three figures are mapped to gray-level images using the same scale. d Shows the independently scaled version of (c). The top left corner of the images corresponds to $(n, m)=(0,0)$. a $\tilde{P}[n, m]$. b $\tilde{P}_{c o n}[n, m]$. c $\tilde{P}_{\text {pro }}[n, m]$. d Independently scaled $\tilde{P}_{\text {pro }}[n, m]$

The performance of the proposed scalar-to-vector wave field mapping is tested by some computer simulations and compared to the performance of the conventional scalar-tovector wave field mapping. In the simulations, the scalar field intensity and the corresponding electric field intensities in space domain are produced. It is observed that the intensity pattern of the scalar field is nearly the same as the intensity pattern of the corresponding electric field in 
different frequency regions. However, if the conventional scalar-to-vector wave field mapping is used, the difference between the corresponding intensity patterns can be quite large. In this respect, the power equalizing scalarto-vector wave field mapping should be preferred over the conventional mapping; this is particularly important in some applications, such as in wide-angle imaging or optical communications.

\section{References}

1. Andrews, H.C., Hunt, B.R.: Digital Image Restoration. PrenticeHall Inc., New Jersey (1977)

2. Balanis, C.A.: Antenna Theory: Analysis and Design. Wiley, New Jersey (2005)

3. Borgiotti, G.: Fourier transforms method in aperture antennas problems. Alta Frequenza 32, 196-204 (1963)

4. Born, M., Wolf, E.: Principles of Optics: Electromagnetic Theory of Propagation, Interference and Diffraction of Light. Cambridge University Press, Cambridge (1999)

5. Bouwkamp, C.J.: Diffraction theory. Rep. Prog. Phys. 17, 35-100 (1954)

6. Cannon, T.M.: Digital image deblurring by nonlinear homomorphic filtering. Ph.D. thesis, Computer Science Department, University of Utah (1974)

7. Delen, N., Hooker, B.: Free-space beam propagation between arbitrarily oriented planes based on full diffraction theory: a fast Fourier transform approach. J. Opt. Soc. Am. A 15, 857-867 (1998)

8. Erdélyi, A., Magnus, W., Oberhettinger, F.: Tables of Integral Transforms. McGraw-Hill, New York (1954)

9. Esmer, G.B.: Computation of holographic patterns between tilted planes. Master's thesis, Bilkent University (2004)

10. Esmer, G.B., Onural, L., Ozaktas, H.M.: Exact diffraction calculation from fields specified over arbitrary curved surfaces. Opt. Commun. 284, 5537-5548 (2011)

11. Esmer, G.B., Uzunov, V., Onural, L., Ozaktas, H.M., Gotchev, A.: Diffraction field computation from arbitrarily distributed data points in space. Signal Process. Image Commun. 22, 178-187 (2007)

12. Gabor, D.: A new microscopic principle. Nature 161, 777-778 (1948)

13. Geng, J.: Three-dimensional display technologies. Adv. Opt. Photon. 5, 456-535 (2013)

14. Goodman, J.W.: Introduction to Fourier Optics. McGraw-Hill, New York (1996)

15. Grigoryan, A.M., Doughelly, E.R.: Optimization of linear filters under power-spectral-density stabilization. IEEE Trans. Signal Process. 49, 2292-2300 (2001)

16. Grigoryan, A.M., Dougherty, E.R., Agaian, S.S.: Optimal Wiener and homomorphic filtration: review. Signal Process. 121, 111-138 (2016)

17. Kong, J.A.: Electromagnetic Wave Theory. Wiley, New Jersey (1986)

18. Kulce, O., Onural, L., Ozaktas, H.M.: Evaluation of the validity of the scalar approximation in optical wave propagation using a systems approach and an accurate digital electromagnetic model. J. Mod. Opt. 63, 2382-2391 (2016)

19. Leith, E.N., Upatnieks, J.: Reconstructed wavefronts and communication theory. J. Opt. Soc. Am. 52, 1123-1130 (1962)

20. Lueder, E.: 3D Displays. Wiley, New York (2012)

21. Luneburg, R.K., Herzberger, M.: Mathematical Theory of Optics. University of California Press, Los Angeles (1964)
22. Onural, L.: Some mathematical properties of the uniformly sampled quadratic phase function and associated issues in digital Fresnel diffraction simulations. Opt. Eng. 43, 2557-2563 (2004)

23. Onural, L., Yaras, F., Kang, H.: Digital holographic threedimensional video displays. Proc. IEEE 99, 576-589 (2011)

24. Oppenheim, A.V., Schafer, R.W., Buck, J.R.: Discrete-Time Signal Processing. Prentice Hall, New Jersey (1999)

25. Osten, S., Krüger, S., Steinhoff, A.: Spatial light modulators based on reflective micro-displays. Tech. Mess. 73, 149-156 (2006)

26. Rhodes, D.: On the stored energy of planar apertures. IEEE Trans. Antennas Propag. 14, 676-683 (1966)

27. Şahin, E., Onural, L.: Scalar diffraction field calculation from curved surfaces via Gaussian beam decomposition. J. Opt. Soc. Am. A 29, 1459-1469 (2012)

28. Şahin, E., Onural, L.: Calculation of the scalar diffraction field from curved surfaces by decomposing the three-dimensional field into a sum of Gaussian beams. J. Opt. Soc. Am. A 30, 527-536 (2013)

29. Sherman, G.C.: Application of the convolution theorem to Rayleigh's integral formulas. J. Opt. Soc. Am. 57(4), 546-547 (1967)

30. Theimer, O., Wassermann, G.D., Wolf, E.: On the foundation of the scalar diffraction theory of optical imaging. Proc. Roc. Soc. A 212, 426-437 (1952)

31. Yaras, F., Kang, H., Onural, L.: State of the art in holographic displays: a survey. J. Disp. Technol. 6, 443-454 (2010)

32. Yeh, P., Gu, C.: Optics of Liquid Crystal Displays. Wiley, New York (1999)

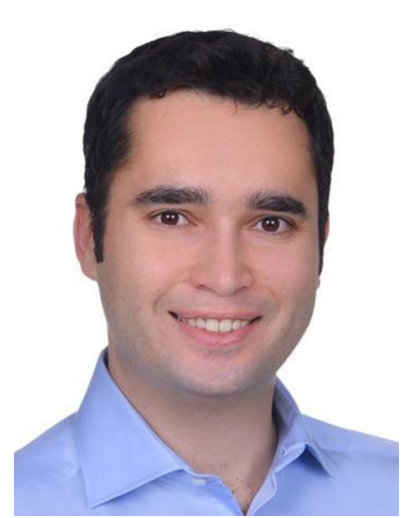

Onur Kulce received his B.S. and M.S. degrees from Electrical and Electronics Engineering, Bilkent University, Ankara, Turkey, in 2010 and 2012, respectively. $\mathrm{He}$ is currently pursuing his Ph.D. degree in the same department. His research interests include signal processing for scalar and polarized optical wave fields and its applications to holographic 3DTV. He was a TUBITAK scholar and worked as a research and teaching assistant in Electrical and Electronics Engineering at Bilkent University between 2010 and 2017. Since 2017, he is working as a research and development engineer in Communications and Spectrum Management Research Center (ISYAM) at Bilkent University.

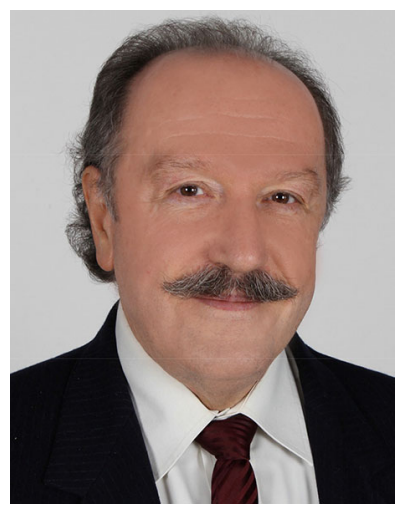

Levent Onural received his Ph.D. degree in electrical and computer engineering from State University of New York at Buffalo in 1985; his B.S. and M.S. degrees are from METU in 1979 and 1981, respectively. He was a Fulbright scholar between 1981 and 1985 . He joined the Electrical and Electronics Engineering Department of Bilkent University, Ankara, Turkey, in 1987, where he is a full professor at present; he served as the Dean of Engineering and Head of the Graduate School of 
Engineering and Science at Bilkent University between 2010 and 2016. His current research interests are in the area of image and video processing, with emphasis on video coding, 3DTV, holographic 3DTV and signal processing aspects of optical wave propagation. He was the coordinator of European Union funded 3DTV Project (20042008). He served as the co-leader of the 3D Media Cluster, which is an umbrella organization formed by many European Union-funded 3D-related projects (2008-2011). He was a member of the board of European Union funded Real 3D Project (2008-2011) which focused on fundamentals of end-to-end holographic 3D imaging systems. Dr. Onural received an incentive award from TUBITAK of Turkey in 1995. He also received a Third Millennium Medal from IEEE in 2000. Dr. Onural is a fellow of IEEE. He served IEEE as the Director of IEEE Region 8 (Europe, Middle East and Africa) in 20012002, as the Secretary of IEEE in 2003. He was a member of IEEE Board of Directors (2001-2003), IEEE Executive Committee (2003) and IEEE Assembly (2001-2002). He received IEEE Haraden Pratt Award, EURASIP Group Technical Achievement Award and Bilkent University Distinguished Teacher Award, all in 2011. 